Editorial

\title{
Fundaciones para la investigación biomédica hospitalaria
}

La investigación biomédica es un área más del desarrollo tecnológico y científico actual que, de una forma amplia, produce y aporta los conocimientos científicos en los que se sustenta la asistencia clínica y el armamentarium terapéutico.

La diversidad y complejidad de este tipo de investigación necesita de un soporte específico en varios campos. Aun siendo el mas importante de todos ellos el de la financiación, la gestión de la investigación ha alcanzado un alto grado de especialización para adecuarse a sus necesidades y características propias.

La investigación biomédica y biotecnológica pueden tener, y de hecho tienen, diferentes escenarios. Uno de ellos, el hospitalario, se viene desarrollando como una parte integral de la actividad de los centros, estando reconocida esta actividad en la Ley General de Sanidad de 1986, desde cuyo articulado se fomenta como un medio para mejorar el sistema sanitario. Así pues, en la práctica tenemos, de forma real, áreas de investigación implantadas dentro de los centros sanitarios, que han de conjugar su funcionamiento administrativo y científico con el de la gestión sanitaria.

Se podría considerar, desde esta óptica de la gestión sanitaria, que la gestión de la investigación biomédica reune unas características y necesidades que no pueden ser manejadas de forma óptima desde la administración hospitalaria de nuestro sistema sanitario público. Durante la década pasada se fueron desarrollando otros modelos de gestión que si bien en los centros privados se aplicaron a la práctica con escasa demora, en el sistema sanitario público su aplicación se vio ralentizado por la tramitación habitual en la administración pública. Estos modelos de gestión propios de la investigación biomédica, acabaron concretándose en las Fundaciones para la Investigación Biomédica de los Hospitales públicos.

La estructura organizativa de las Fundaciones Biomédicas es similar a la del resto de Fundaciones, se rigen por un Patronato y su correspondiente Presidente, un Director, que puede tener diferente perfil, entre puramente gestor a científico u otras formas intermedias o combinadas, y dos órganos asesores, la Comisión Científica, que deberá asumir la elaboración de propuestas sobre la política científica de la Fundación, y el Consejo Asesor que tendría su cometido, en el asesoramiento al Patronato en todos aquellos temas mas dirigidos a la política económica y social de la entidad. A su vez, las Fundaciones tienen un órgano rector, que vela por su correcto funcionamiento, que es el Protectorado de Fundaciones de cada Comunidad Autónoma.

Estas Fundaciones, hoy implantadas en muchos centros hospitalarios, han asumido, como entidades jurídicas independientes, la gestión de la investigación básica, aplicada y clínica que se desarrolla en estos centros. Sin embargo, su propia estructura permite ir más allá de la pura gestión. Las Fundaciones tienen capacidad para impulsar y fomentar la investigación, esto lo pueden conseguir de una forma indirecta con la simple realización de una gestión aplicada, y de una forma directa, que es la que aquí quiero resaltar, mediante la generación de recursos propios que les permitirá abordar y desarrollar programas científicos que acabarán revirtiendo su beneficio en la propia entidad.

La creación y puesta en funcionamiento de las Fundaciones hospitalarias es, sin lugar a dudas, un apoyo de gran valor para una de las tres finalidades que se les asigna a los hospitales, curar, enseñar e investigar. En nuestros hospitales la investigación, clásicamente, ha sido la labor menos favorecida, sin embargo, una de sus ramas, la investigación clínica, que constituye el único método racional para el desarrollo final de un nuevo fármaco, es patrimonio exclusivo de los hospitales y su gestión, regida en la actualidad por un marco legal que está lejos de ser el idóneo, se puede ver beneficiada por el soporte que le ofrecen las Fundaciones. Pero el área 


\section{F. Bonilla}

de la investigación biomédica que meridianamente se ve favorecida es la investigación básica. La epidémica escasez de asignación de recursos a este fin en nuestro país, multiplica los esfuerzos de los investigadores para conseguirlos y para llevar a cabo la tramitación de los mismos. Las Fundaciones suponen una ayuda en estos aspectos burocráticos y al mismo tiempo, como ya hemos comentado, convertirse en una fuente propia de financiación al servicio de la investigación.

En conclusión, las Fundaciones Biomédicas no van a generar per se un incremento espectacular de la producción científica hospitalaria, si no existe un potencial humano con capacidad creativa y con espíritu de dedicación a la investigación, además de contar con la imprescindible financiación, pero indiscutiblemente son una herramienta incorporada dentro de un sistema de gestión que no podía ofrecer la mejor solución a las situaciones específicas requeridas por la misma, y que facilitan y potencian la actividad investigadora en los hospitales, ayudando a su aplicabilidad y, en suma, contribuyendo a mejorar la calidad asistencial.

Félix Bonilla

Editor Asociado Revista Oncología Director de la Fundación para la Investigación Biomédica Hospital Universitario Puerta de Hierro

Madrid 